\title{
Thiopurine methyltransferase activity in children with acute myeloid leukemia
}

\author{
JOANNA SOBIAK $^{1 *}$, JOLANTA SKALSKA-SADOWSKA $^{2 *}$, MARIA CHRZANOWSKA $^{1}$, \\ MATYLDA RESZTAK $^{1}$, SYLWIA KOŁTAN ${ }^{3}$, MARIUSZ WYSOCKI ${ }^{3}$ and JACEK WACHOWIAK ${ }^{2}$ \\ ${ }^{1}$ Department of Physical Pharmacy and Pharmacokinetics, Poznan University of Medical Sciences, 60-781 Poznań; \\ ${ }^{2}$ Department of Pediatric Oncology, Hematology and Transplantology, Poznan University of Medical Sciences, \\ 60-572 Poznań; ${ }^{3}$ Department of Pediatrics, Hematology and Oncology, Collegium Medicum, \\ Nicolaus Copernicus University, 85-094 Bydgoszcz, Poland
}

Received October 15, 2017; Accepted April 16, 2018

DOI: $10.3892 /$ ol.2018.9191

\begin{abstract}
Activity of the enzyme thiopurine methyltransferase (TPMT) determines the anti-leukemic effect of thiopurines used in the chemotherapy of acute lymphoblastic leukemia (ALL) and acute myeloblastic leukemia (AML). TPMT status and its effects on treatment outcome have been studied extensively in ALL and autoimmune disorders, but few data is available on TPMT in AML. The present study assessed the genetic polymorphisms and activity of TPMT in children with AML at different treatment stages, and compared the results with those obtained for children with ALL. The study included 33 children with AML (0.7-19.7 years) treated with 6-thioguanine (6-TG) according to the AML-BFM 2004 Protocol. Blood samples were collected at diagnosis, during and following maintenance chemotherapy from 8,10 and 17 patients with AML (the assay was performed at two time points in 2 patients), respectively. Blood samples from 105 children with ALL were obtained at diagnosis, during the maintenance chemotherapy and following the cessation of the chemotherapy from 16, 55 and 34 children, respectively. The activity of TPMT in red blood cells lysates was measured using an enzymatic reaction based on the conversion of 6-mercaptopurine into 6-methylmercaptopurine, involving S-adenozyl-L-methionine as the methyl group donor. TPMT mutations were determined using a polymerase chain reaction/restriction fragment length polymorphism method. Median TPMT activity at diagnosis, during maintenance chemotherapy and following chemotherapy was 43.1, 47,3 and $41.7 \mathrm{nmol} 6-\mathrm{mMP} \mathrm{g}^{-1} \mathrm{Hb} \mathrm{h}^{-1}$, respectively. All
\end{abstract}

Correspondence to: Mrs. Joanna Sobiak, Department of Physical Pharmacy and Pharmacokinetics, Poznan University of Medical Sciences, 6 Święcickiego, 60-781 Poznań, Poland

E-mail: jsobiak@ump.edu.pl

*Contributed equally

Key words: thiopurine methyltransferase, acute myeloid leukemia, children patients with AML exhibited the homozygous TPMT*1/*1 genotype, with the exception of 1 , who was a heterozygote with the TPMT $1 /{ }^{*} 3 \mathrm{C}$ genotype and demonstrated a TPMT

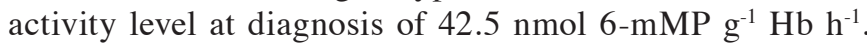
At each chemotherapy stage, the median TPMT activities in children with AML were significantly increased compared with the median TPMT activities in children with ALL. The preliminary results suggest that the TPMT activity in AML may be increased compared with that in ALL. Comprehensive studies on the association between thiopurine metabolism and treatment outcome in AML are required, with regard to the cytogenetic and molecular factors currently used for AML risk stratification.

\section{Introduction}

Acute leukemia is the most common type of pediatric malignancy, of which $80 \%$ cases are classified as acute lymphoblastic leukemia (ALL) and 15-25\% as acute myeloblastic leukemia (AML) $(1,2)$. There is a significant discrepancy in the outcomes of pediatric ALL and AML, with 5-year survival of $90 \%$ in ALL compared with $70 \%$ in AML $(1,3,4)$. The difficulties in AML treatment include limited therapeutic options and multi-drug resistance exhibited by AML myeloblasts, despite the identification of prognostic markers (5).

One of the drugs used for the treatment of children with AML is 6-thioguanine (6-TG), administered during maintenance chemotherapy at a dose of $40 \mathrm{mg} / \mathrm{m}^{2} / 24 \mathrm{~h}$ for 1 year (AML-BFM 2004 Protocol) (6). 6-TG and 6-mercaptopurine (6-MP) belong to the thiopurine drug family, which includes purine analogs with anticancer and immunosuppressive activities. All thiopurines are prodrugs, and must be converted in vivo into thioguanine nucleotides (TGNs), which are incorporated into DNA to exert a cytotoxic effect. 6-TG is converted directly in a single-step pathway, while 6-MP requires a three-step conversion. The major competing metabolic process is S-methylation, catalyzed by thiopurine methyltransferase (TPMT). 6-MP is methylated to methylMP and 6-TG to methylTG. The intermediates of the parent drugs may also be methylated: 6-thioinosine 5'-monophosphate (TIMP) to methyl-TIMP (MeTIMP) and thioguanine monophosphate 
(TGMP) to methyl-TGMP (MeTGMP) (7) (Fig. 1). MeTIMP inhibits de novo purine synthesis (DNPS) and contributes to the cytotoxic effect of 6-MP in addition to TGN formation. Although $>1$ of the thiopurine metabolites may inhibit DNPS, MeTIMP is a more potent inhibitor compared with the others, including MeTGMP (7-10).

TPMT activity determines the availability of thiopurines in the conversion to TGNs (8). It follows a trimodal pattern of distribution controlled by genetic polymorphisms, with TPMT ${ }^{\mathrm{L}}$ (low-activity variant alleles $\mathrm{TPMT}^{*} 2$-*38) or $^{*}$ $\mathrm{TPMT}^{\mathrm{H}}$ (high-activity wild-type allele TPMT*1) at a single genetic locus. Overall, $0.3 \%$ of the Caucasian population are TPMT-deficient (homozygous TPMT $^{\mathrm{L}} / \mathrm{TPMT}^{\mathrm{L}}$ genotype), $11 \%$ exhibit intermediate TPMT activity (heterozygous $\mathrm{TPMT}^{\mathrm{L}} / \mathrm{TPMT}^{\mathrm{H}}$ genotype) and $89 \%$ exhibit high TPMT activity $\left(\mathrm{TPMT}^{\mathrm{H}} / \mathrm{TPMT}^{\mathrm{H}}\right.$ genotype) $(9,11,12)$. At present, the reason for the unequal distribution of TPMT activity in the intermediate- and high-activity groups remains unknown.

TPMT activity is associated with sensitivity and toxicity to thiopurines within individuals (11). People who are homozygous for nonfunctional TPMT alleles (TPMT ${ }^{\mathrm{L}} / \mathrm{TPMT}^{\mathrm{L}}$ genotype) develop severe and potentially fatal 6-MP-induced hematologic toxicity (11).

TPMT status and its implications in treatment outcome have been studied extensively in ALL and autoimmune disorders (13-16), but little data is available on TPMT in AML $(3,10)$. As a preliminary evaluation to compare TPMT status in AML with the one in ALL, we report the results of TPMT genotype and phenotype assessment in children treated for AML.

\section{Materials and methods}

Patients. The present study included 33 children (18 males and 15 females), aged between 0.7-19.7 years (mean age, 10.3 years), treated for AML according to the control arm of AML-BFM 2004 Protocol (6). Patients were recruited between January 2007 to December 2015 from the Department of Pediatric Oncology, Hematology and Transplantology, Karol Jonscher Clinical Hospital, Poznań and the Department of Pediatrics, Hematology and Oncology, Antoni Jurasz University Hospital, Bydgoszcz. The characteristics of the children in the study group are summarized in Table I. Based on the protocol risk group assignments, the chemotherapy used in the standard risk (SR) arm consisted of four cycles of intensive chemotherapy (AIE: Cytarabine, $100 \mathrm{mg} / \mathrm{m}^{2} /$ day $48 \mathrm{~h}$ continuous-infusion on days $1-2$ and $100 \mathrm{mg} / \mathrm{m}^{2}$ twice a day on days $3-8$, idarubicin $12 \mathrm{mg} / \mathrm{m}^{2} /$ day on days 3,5 and 7, etoposide $150 \mathrm{mg} / \mathrm{m}^{2} /$ day on days 6-8; AI: Cytarabine $500 \mathrm{mg} / \mathrm{m}^{2} /$ day $96 \mathrm{~h}$ continuous-infusion days $1-4$, idarubicin $7 \mathrm{mg} / \mathrm{m}^{2} /$ day, days 3,5 ; hAM: Cytarabine $1 \mathrm{~g} / \mathrm{m}^{2}$ twice a day, on days 1,2 and 3 , mitoxantrone $10 \mathrm{mg} / \mathrm{m}^{2} /$ day days 3 and 4 ; HAE: Cytarabine $3 \mathrm{~g} / \mathrm{m}^{2}$ twice a day, on days 1,2 and 3 , etoposide $125 \mathrm{mg} / \mathrm{m}^{2} /$ day, on days 2, 3, 4 and 5) followed by 1 year maintenance chemotherapy with 6-TG at a dose of $40 \mathrm{mg} / \mathrm{m}^{2} / 24 \mathrm{~h}$. Patients stratified into the high-risk (HR) group received five cycles of intensive chemotherapy (second induction of HAM between AIE and AI: Cytarabine $3 \mathrm{~g} / \mathrm{m}^{2}$ twice a day on days 1,2 and 3; mitoxantrone $10 \mathrm{mg} / \mathrm{m}^{2} /$ day on days 3 and 4) and either proceeded to hematopoietic stem cell transplantation (HSCT) if a well-matched familiar donor was

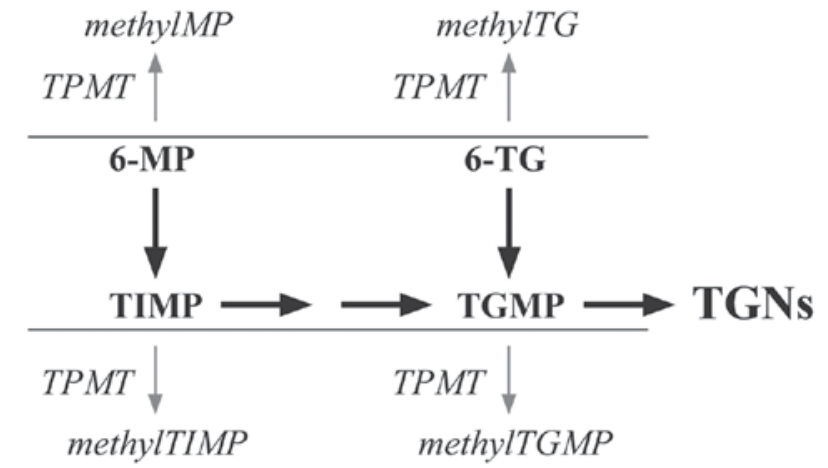

Figure 1. Simplified schematic diagram of the primary metabolic pathways of 6-MP and 6-TG. methylMP, methylmercaptopurine; methylTG, methylthioguanine; methylTGMP, methylthioguanosine monophosphate; methylTIMP, methylthioinosine monophosphate; TGMP, thioguanosine monophosphate; TGNs, thioguanine nucleotides; TIMP, thioinosine 5'-monophosphate; TPMT, thiopurine methyltransferase; 6-MP, mercaptopurine; 6-TG, thioguanine.

available, or received the same maintenance chemotherapy as the SR group. The intensive chemotherapy treatment did not include 6-TG. Only patients in remission were treated with maintenance chemotherapy. None of the patients included developed any significant infection during maintenance treatment. In total, 29 children included in the AML study group were treated with 6-TG. Within the time-frame of the present study, the intended evaluation of TPMT consecutive activity at different stages of treatment in the same AML patients appeared impossible due to the following causes: Admission to the oncology clinic shortly following red blood cell (RBC) transfusion; transfusion-dependence during intensive chemotherapy; transfer for HSCT; and relapses and referrals to external clinics for follow-up subsequent to intensive chemotherapy. TPMT evaluations were performed using blood samples collected at diagnosis from 8 patients and during and following maintenance chemotherapy from 10 and 17 patients. The two latter subgroups included the only 2 patients in whom the assay was performed at both these stages of AML. Samples were taken at least 30 days following the beginning of treatment and at least 6 weeks following the cessation of maintenance chemotherapy. The present study was approved by the Bioethical Committee at Poznan University of Medical Sciences (Poznań, Poland), was performed in accordance with the 1964 Declaration of Helsinki and its later amendments. Written informed consent was obtained from the parents of the patients prior to initiating the study.

Blood samples from 105 children with ALL were obtained at: Point of diagnosis ( 16 children, 8 males and 8 females, aged 2-16 years, mean $6.6 \pm 3.5$ years); during the maintenance chemotherapy (55 children, 28 males and 27 females, aged 1-17 years, mean $6.9 \pm 4.2$ years); and following the cessation of the chemotherapy ( 34 children, 16 males and 18 females, aged 9-28 years, mean $16 \pm 4$ years). The children with ALL were treated according to the ALL IC-BFM 2002 Chemotherapy Treatment protocol (17), including maintenance treatment with 6-MP $\left(50 \mathrm{mg} / \mathrm{m}^{2} /\right.$ day $)$ and methotrexate $\left(20 \mathrm{mg} / \mathrm{m}^{2} /\right.$ week $)$. The data were described previously (18).

TPMT phenotype assays were performed at least 8 weeks after the most recent blood transfusion. RBC lysates were obtained from the venous blood of patients with AML and 
Table I. Clinical characteristics of children and adolescents with acute myeloblastic leukemia.

\begin{tabular}{|c|c|}
\hline Characteristics & Value $^{a}$ \\
\hline \multicolumn{2}{|l|}{ Sex } \\
\hline Male & 18 \\
\hline Female & 15 \\
\hline Age, years (mean) & $0.7-19.7(10.3)$ \\
\hline WBC at diagnosis, median (range) & $14.0(0.3-282.0)\left[\mathrm{x} 10^{9} / 1\right]$ \\
\hline$<3 \times 10^{9} / 1$ & 8 \\
\hline $3-15 \times 10^{9} / 1$ & 10 \\
\hline$>15-100 \times 10^{9} / 1$ & 9 \\
\hline$>100 \times 10^{9} / 1$ & 6 \\
\hline Platelets at diagnosis, median (range) & $35(2-244)\left[\times 10^{9} / 1\right]$ \\
\hline$\%$ of blasts in bone marrow at diagnosis, median (range) & 66 (range $25-90$ ) \\
\hline \multicolumn{2}{|l|}{ Molecular cytogenetics data } \\
\hline FLT3 ITD.+ & 1 \\
\hline $\mathrm{t}(15 ; 17)$ & 3 \\
\hline $\mathrm{t}(8 ; 21)$ & 3 \\
\hline Other karyotype abnormalities & 4 \\
\hline $47, \mathrm{XY},+8, \mathrm{t}(9 ; 11)(\mathrm{p} 22 ; \mathrm{q} 23)$ & 1 \\
\hline 90-92,XXYY,der(4) & 1 \\
\hline $47 X X,+8[10] / 46 X X[8]$ & 1 \\
\hline $46, X Y, t(8 ; 3 ; 21)(q 22 ; ? q 25 ; q 22), \operatorname{del}(9)(q 21 q 22)$ & 1 \\
\hline Normal karyotype & 13 \\
\hline Data unavailable & 9 \\
\hline Treated with chemotherapy alone including 6-TG & 29 \\
\hline Treated with chemotherapy and HSCT without 6-TG & 4 \\
\hline WBC at the end of chemotherapy, median (range) & $4.3(2-8)\left[\times 10^{9} / 1\right]$ \\
\hline Proportion of CR1, n (\%) & $33(100)$ \\
\hline Relapse, n (\%) & $8(24)$ \\
\hline Succumbed in progression of leukemia, $\mathrm{n}(\%)$ & $6(18)$ \\
\hline EFS, years (mean \pm SEM) & $4(75 \pm 7 \%)$, median follow up 51.8 months \\
\hline OS, years (mean \pm SEM) & $4(78 \pm 7 \%)$, median follow up 76.1 months \\
\hline
\end{tabular}

${ }^{a}$ Values indicate the number of patients unless stated otherwise. WBC, white blood cell count; 6-TG, thioguanine; HSCT, hematopoietic stem cell transplantation; EFS, probability of event-free survival; OS, probability of overall survival; SEM, standard error of the mean.

healthy adult volunteers. For the ALL study, the control group were 39 healthy children aged 2-15 years (mean $6.9 \pm 4.0$ years). Among the control group, there were 18 males aged 2-15 years (mean $7.3 \pm 3.9$ years) and 21 females aged 2-14 years (mean 6.7 \pm 4.1 years). These participants were recruited in (January-December) 2005 year in the Outpatient Clinic for Proliferative Diseases (Karol Jonscher Clinical Hospital, Poznań) for other than haematological indications. For the AML study, the control group consisted of the control group from ALL study and an additional 5 healthy adult volunteers aged 25-60 years (mean 36 \pm 14 years; 2 men and 3 women) who were recruited in Regional Centre of Blood Donation and Blood Treatment in Poznań. Blood samples $(1 \mathrm{ml})$ were collected into EDTA tubes and centrifuged for $10 \mathrm{~min}$ at $1,700 \mathrm{x} \mathrm{g}$ at room temperature. The plasma was removed and RBCs were washed with isotonic salt solution $(0.9 \% \mathrm{NaCl}$; Polfa Lublin, Poland), and re-centrifuged for $10 \mathrm{~min}$ at $1,700 \mathrm{x} \mathrm{g}$ at room temperature. Samples of concentrated RBC were then stored at $-80^{\circ} \mathrm{C}$ until analysis was performed. After thawing, lysates were diluted using isotonic salt solution (1:1) and centrifuged at room temperature for $10 \mathrm{~min}$ at $1,300 \mathrm{x}$ g to remove $\mathrm{RBC}$ membranes. TPMT activity and haemoglobin concentration were determined in the same sample of RBC lysate. The activity of TPMT was measured in RBC lysates using an enzymatic reaction, described previously $(19,20)$, based on the conversion of the substrate, 6-MP, into 6-methylmercaptopurine (6-mMP), involving S-adenozyl-L-methionine (SAM) as the methyl group donor. For the calibration curves (generated using MS Excel 2010; Microsoft Corporation, Redmond, WA, USA), the solutions consisted of different final 6-mMP concentrations (3.1-75.0 ng/50 $\mu \mathrm{l} \mathrm{RBC}$ lysates), 0.2 M potassium dihydrogenphosphate and standard RBC lysates were used. For determination of the TPMT activity, $50 \mu 1 \mathrm{RBC}$ lysates were mixed with 6-MP solution (500 mg/l), SAM $(640 \mu \mathrm{mol} / \mathrm{l})$ and 
$0.1 \mathrm{M}$ potassium dihydrogenphosphate. Following $1 \mathrm{~h}$ incubation at $37^{\circ} \mathrm{C}, 6-\mathrm{mMP}$ was extracted in the non-polar phase, and then the 6-mMP plasma concentration was determined using the high-performance liquid chromatography (HPLC) method (20) with UV detector (at $290 \mathrm{~nm}$ ). The flow rate was $1 \mathrm{ml} / \mathrm{min}$ and $30 \mu \mathrm{l}$ was injected into HPLC for analysis. The calibration curves were expressed as the association between the peak surface area determined in samples and 6-mMP concentration expressed as ng/50 $\mu \mathrm{l} \mathrm{RBC}$ lysates. In the same RBC lysate sample, hemoglobin concentration was determined using the cyanmethemoglobin method (Stamar; Department of Reagents for Laboratory Diagnositics; Dąbrowa Górnicza, Poland). Hemoglobin concentration was determined using the spectrophotometric method with Drabkin's reagent (Stamar; Department of Reagents for Laboratory Diagnositics; Dąbrowa Górnicza, Poland; https://stamar.pl/). According to the manufacturers protocol, the concentration of potassium ferricyanide and potassium cyanide were 0.6 and $0.7 \mathrm{nM}$, respectively. To determine the concentration of hemoglobin in patient samples, $10 \mu \mathrm{l}$ RBC lysate was mixed with $2.5 \mathrm{ml}$ Drabkin's reagent. Following after $3 \mathrm{~min}$, the absorbance of the solution was determined at a wavelength of $540 \mathrm{~nm}$. Additionally, blind, control and standard samples were prepared according to the manufacturer's protocol. A volume of $10 \mu \mathrm{l}$ of purified water, $\mathrm{RBC}$ from control groups and cyanmethemoglobin standard solution was added into the tubes containing $2.5 \mathrm{ml}$ of Drabkin's reagent for blind, control and standard samples, respectively. TPMT activity was expressed as nmol of produced $6-\mathrm{mMP} / \mathrm{g}$ $\mathrm{Hb} / \mathrm{h}$. All calculations were performed using MS Excel 2010 (Microsoft Corporation, Redmond, WA, USA). Samples from each patient were examined twice.

TPMT mutations $\left(\mathrm{TPMT}^{*} 2, \mathrm{TPMT}^{*} 3 \mathrm{~A}, \mathrm{TPMT}^{*} 3 \mathrm{~B}\right.$ and TPMT $^{*} 3 \mathrm{C}$ ) and the wild type TPMT 1 were determined with a polymerase chain reaction/restriction fragment length polymorphism method (21) at the Department of Experimental and Clinical Pharmacology, Pomeranian Medical University (Szczecin, Poland).

Statistical analysis. The data are presented as the median and range. Although some data was normally distributed, the median was presented to enable clear comparisons of data in subgroups. Statistical analysis was performed using Statistica software version 12.0 (StatSoft, Inc., Tulsa, OK, USA). P<0.05 was considered to indicate a statistically significant difference. Normality was determined using the Kolmogorov-Smirnov test for groups $>50$ patients (all ALL patients assessed during maintenance chemotherapy) or the Shapiro-Wilk test for the remaining groups which were $<50$ patients. All differences between groups were estimated using a Student's t-test (for normally distributed data) and a Mann-Whitney or Kruskal-Wallis tests for non-normally distributed data. TPMT activity was normally distributed in males and females with AML, at the time of AML diagnosis, during treatment of AML and in all groups with ALL. Non-normal distributions were observed for TPMT activity in the entire AML group and among children following AML treatment. In the case of unequal variations, Cochran's $\mathrm{C}$ test was applied. The Kaplan-Meier method was also used for the relapse risk analysis and log rank test for the comparative analysis. The association between TPMT activity and rank variables was assessed with Spearman's rank correlation coefficient. The difference in the distribution of TPMT alleles between AML and ALL groups was estimated using the z-test for the difference of proportions.

\section{Results}

In children with AML, the levels of TPMT activity varied between 25.9-91.6 nmol 6-mMP g ${ }^{-1} \mathrm{Hb} \mathrm{h}^{-1}$, with median $43.6 \mathrm{nmol} 6-\mathrm{mMP} \mathrm{g}^{-1} \mathrm{Hb} \mathrm{h}^{-1}$.

Median TPMT activity in the children with AML at the point of diagnosis was $43.1 \mathrm{nmol} 6-\mathrm{mMP} \mathrm{g}^{-1} \mathrm{Hb} \mathrm{h}^{-1}$. During maintenance chemotherapy, median TPMT activity was $47.3 \mathrm{nmol} 6-\mathrm{mMP} \mathrm{g}{ }^{-1} \mathrm{Hb} \mathrm{h}^{-1}$. Following the cessation of chemotherapy, the median TPMT activity was $41.72 \mathrm{nmol}$ 6-mMP g-1 $\mathrm{Hb} \mathrm{h}^{-1}$.

All children with AML exhibited the TPMT*1/*1 genotype, with the exception of 1 , who was a heterozygote with the genotype TPMT ${ }^{*} 1 /{ }^{*} 3 \mathrm{C}$. TPMT activity in this child was determined at diagnosis, and measured $42.5 \mathrm{nmol}$ 6-mMP g ${ }^{-1} \mathrm{Hb} \mathrm{h}^{-1}$.

No statistical differences in TPMT activity were observed with respect to age $\left(r_{s}=0.001, P=0.997\right.$ in the whole group; $r_{s}=-0.733, P=0.695$ among 29 children treated with 6-TG) or sex $(\mathrm{P}=0.677)$. The TPMT activity was similar regardless of whether the assay was performed at diagnosis, during or following maintenance chemotherapy $(\mathrm{P}=0.919)$. The TPMT activity results are detailed in Table II. Overall, two consecutive TPMT evaluations during and following chemotherapy for 2 children also revealed convergent results $(50.9$ and $52.5 \mathrm{nmol}$ 6- $\mathrm{mMP} \mathrm{g}^{-1} \mathrm{Hb} \mathrm{h}^{-1}$ for the first child, and 34.6 and $34.9 \mathrm{nmol}$ 6-mMP g ${ }^{-1} \mathrm{Hb} \mathrm{h}^{-1}$ for the second child).

Subsequent to a median follow-up period of 4.3 years (range, 0.3-17.0 years), 8/33 patients (24\%) relapsed 0.3-3.2 years following diagnosis (median, 1.3 years), with a cumulative risk of relapse $=0.240$. The risk of relapse was not significantly correlated with TPMT activity $\left(r_{s}=-0.002, P=0.912\right.$ in the whole group; $r_{s}=-0.026, P=0.887$ among 29 children treated with 6-TG). The only patient with the heterozygous TPMT genotype relapsed 1.1 year following the first remission; however, the patient's TPMT activity of $42 \mathrm{nmol} 6-\mathrm{mMP} \mathrm{g}^{-1}$ $\mathrm{Hb} \mathrm{h}^{-1}$ assessed at diagnosis did not deviate from the median of the cohort.

In the children with ALL, the median TPMT activity was $30.9 \mathrm{nmol} 6-\mathrm{mMP} \mathrm{g}^{-1} \mathrm{Hb} \mathrm{h}^{-1}$ (range, 6.0-49.5 nmol 6-mMP $\mathrm{g}^{-1} \mathrm{Hb} \mathrm{h}^{-1}$ ); at diagnosis, $25.0 \mathrm{nmol} 6-\mathrm{mMP} \mathrm{g}^{-1} \mathrm{Hb} \mathrm{h}^{-1}$ (range, 6.0-44.0 nmol 6-mMP g ${ }^{-1} \mathrm{Hb} \mathrm{h}^{-1}$ ); during maintenance chemotherapy, $29.7 \mathrm{nmol} 6-\mathrm{mMP} \mathrm{g}^{-1} \mathrm{Hb} \mathrm{h}^{-1}$ (range, 6.6-47.1 nmol 6-mMP $\mathrm{g}^{-1} \mathrm{Hb} \mathrm{h}^{-1}$ ); and following cessation of chemotherapy, $37.3 \mathrm{nmol} 6-\mathrm{mMP} \mathrm{g}^{-1} \mathrm{Hb} \mathrm{h}^{-1}$ (range, 16.3-37.3 nmol 6-mMP g-1 $\left.\mathrm{Hb} \mathrm{h}^{-1}\right)$.

Overall, 1 child with ALL included in the group measured at the point of diagnosis exhibited a heterozygous TPMT*1/*3A genotype. Among the patients with ALL measured during maintenance chemotherapy, 4 were heterozygous ( 3 TPMT $^{*} 1 /^{*} 3 \mathrm{~A}$ and $1 \mathrm{TMPT}^{*} 1 /^{*} 2$ ). The remaining 100 children with ALL were TMPT*1/"1 homozygous.

The proportion of heterozygous patients in the AML group $(3.0 \%, \mathrm{n}=1 / 33)$ did not differ significantly from that in the ALL group $(4.7 \%, \mathrm{n}=5 / 105 ; \mathrm{P}=0.674)$. 
Table II. TPMT activity in association with sex and stage of therapy in all children with acute myeloblastic leukemia, and in the subgroup treated with 6-TG.

A, All studied children $(n=33)$

\begin{tabular}{lc}
\hline Characteristics & TPMT activity (nmol 6-mMP g $\left.\mathrm{Hb} \mathrm{h}^{-1}\right)$ \\
\hline Total, median (range) & $43.7(25.9-91.6)$ \\
Sex, median (range) & \\
Male & $43.5(25.8-84.5)$ \\
Female & $43.6(27.0-91.6)$ \\
Stage of therapy, median (range) & \\
At diagnosis & $43.1(30.6-62.7)$ \\
During maintenance & $47.3(25.9-84.5)$ \\
Following cessation of chemotherapy & $41.7(28.2-91.6)$ \\
\hline
\end{tabular}

B, Children treated with 6-TG $(n=29)$

\begin{tabular}{lc}
\hline Characteristics & TPMT activity (nmol 6-mMP g-1 $\left.\mathrm{Hb} \mathrm{h}^{-1}\right)$ \\
\hline Total, median (range) & $43.3(25.9-91.6)$ \\
Sex, median (range) & $42.5(25.8-84.5)$ \\
Male & $45.2(27.2-91.6)$ \\
Female & \\
Stage of therapy, median (range) & $42.5(30.6-55.8)$ \\
At diagnosis & $43.8(25.9-84.5)$ \\
During maintenance & $41.7(28.2-91.6)$ \\
Following cessation of chemotherapy & 0.964 \\
\hline
\end{tabular}

TMPT, thiopurine methyltransferase.

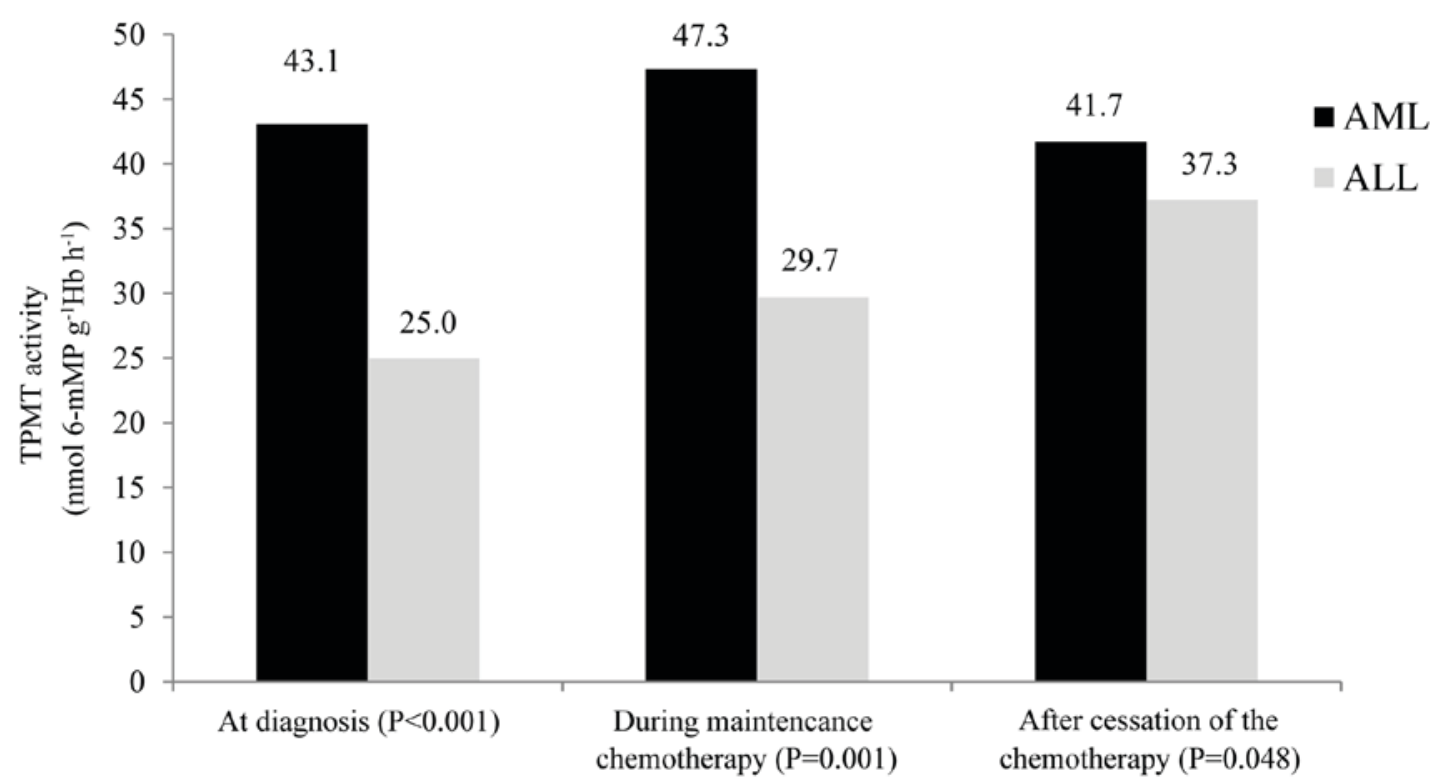

Figure 2. Comparison between median TPMT activity in children with AML (black bar) and ALL (grey bar) at different stages of chemotherapy. AML, acute myeloid leukemia; ALL, acute lymphoblastic leukemia; TPMT, thiopurine methyltransferase.

Median TPMT activities in children with AML at diagnosis, during maintenance chemotherapy and following the cessation of the chemotherapy were increased compared with the median TPMT activities in children with ALL, and the differences were statistically significant $(\mathrm{P}<0.001, \mathrm{P}=0.001$ and $\mathrm{P}=0.048$, respectively) (Fig. 2). 


\section{Discussion}

Variations in TPMT activity according to types of medical condition have been demonstrated in ALL in comparison with inflammatory bowel disease (IBD) and cystic fibrosis $(22,23)$.

In the present study, the median level of TPMT activity in children with AML was significantly increased compared with that in ALL. This did not likely result from the distribution of TPMT alleles, as the proportion of heterozygous patients in the AML group did not differ significantly from that in the ALL group. The results of the present study are in agreement with those of Coulthard et al (10), who also observed increased mean TPMT activity in adult patients with AML, even though TPMT activity was measured in leukemic blasts, whereas it was determined in RBC lysates in the present study. According to Coulthard et al (10), measurements of TPMT activity in $\mathrm{RBC}$ may more accurately reflect its systemic expression in comparison with leukemic blasts. No other studies comparing TPMT activity between patients with AML and ALL were identified during the literature search of the present study.

Anasari et al (24) described the phenomenon of very high TPMT activity was identified in the study of 106 patients with IBD; $\sim 15 \%$ of those who represented high activity demonstrated a value above the reference interval ( $>15$ units $/ \mathrm{ml}$ $\mathrm{RBC}$ ), which was classified as very high. The proportion of ultra-high activity in this group was estimated at 1-2\% $(24,25)$. The same proportion of individuals with ultra-high TPMT activity was determined in a cohort of healthy Caucasian people (25-28). Several candidate factors, including variable number tandem repeats (VNTRs) identified on the TPMT gene promotor, protein kinase $C$, casein kinase substrate in neurons 2 (PACSIN2) and SAM, responsible for the upregulation of TPMT activity were identified by the previous studies $(25,26,29,30)$.

Ultra-high TPMT activity is negatively associated with VNTRs (25). VNTRs modulate TPMT transcription, potentially by altering the spacing between transcription factor binding sites. In particular, the presence of the GCC trinucleotide repeat polymorphism was associated with ultra-high TPMT activity $(25,27)$. The structure of the VNTR region also contributes to the increase in TPMT gene expression observed during maintenance chemotherapy for childhood ALL, presumably due to an interaction between the VNTR genotype and factors associated with the therapy (22). One potential hypothesis is that TPMT gene expression may be high in AML due to the modulation of the VNTR region by an, as of yet, unidentified AML-specific factor.

PACSIN2, a protein subject to genetic polymorphism, is a member of the PACSIN family that regulates cell cycle, endocytosis and autophagy. It has been demonstrated in vitro that individuals with the PACSIN2 rs2413739 CC genotype exhibited higher TPMT activities compared with carriers of other PACSIN2 variants (29). It was also confirmed that PACSIN2 rs2413739 CC-positive patients with ALL exhibited a lower incidence of gastrointestinal and hematological mercaptopurine toxicities in contrast to the ones with $25 \mathrm{CT}$ and TT allele $(29,30)$.

SAM, a methyl donor in reactions catalyzed by TPMT and a number of other methyltransferases, was demonstrated in vitro to be associated with TPMT activity by post-translational stabilization of the TPMT molecule. This effect was confirmed in vivo in $>1,000$ healthy individuals (31). It was realized that the level of SAM was increased in human lung epithelial-like cells exposed to cigarette smoke extract, and that smokers exhibited significantly higher TPMT activity compared with nonsmokers $(26,31)$. Factors affecting SAM biosynthesis exhibited an association with TPMT activity. Methionine, a direct precursor of SAM, is an essential dietary amino acid and is regenerated from homocysteine and 5-methyltetrahydrofolate (5-Me-THF) (26). 5-Me-THF is a final product of the folate cycle and its activity depends on folate intake and the activity of folate-metabolizing enzymes including methylene tetrahydrofolate reductase (MTHFR). A positive association between folate and SAM levels was indicated in a mathematical model of the methionine cycle, which predicted a rise in SAM levels due to increase of 5-Me-THF. MTHFR activity is modulated by MTHFR gene polymorphisms, with a $50 \%$ reduction in homozygosity for its $677 \mathrm{C}>\mathrm{T}$ variant exhibited by $25-43 \%$ of population (26). Individuals with decreased MTHFR activity exhibit lower serum folate level, reduced homocysteine-to-methionine regeneration and lower SAM synthesis, but adequate folate intake may reduce this effect (26). The expression of factors, including PACSIN2, MTHFR, folates, methionine and SAM may contribute to different TPMT activity in ALL as compared with AML. It was demonstrated that the inhibition of DNPS by MeTIMP resulted in a decreased concentration of ATP, which impairs SAM biosynthesis (32). Therefore, the lack of MeTIMP formed exclusively with 6-MP leads to increased SAM (32). This may be in concordance with the hy pothesis of Coulthard et al (10), which suggested that higher TPMT activity was observed in patients with AML as they were not treated with 6-MP (10). However, Lennard et al (33) did not identify a difference in TPMT activity in children treated for ALL with 6-MP compared with those treated with 6-TG.

Lower TPMT activity in ALL in comparison with AML may be associated with co-administration of methotrexate, which was identified to bind to TPMT and inhibit its activity (31). Variety in TPMT activity due to ethnicity, RBC lifespan and kidney function has also been demonstrated (13). The effects of other untested or unknown TPMT gene allelic variants in the results of the present study cannot be completely excluded; however, they occur very rarely (13).

The results of the present study may have clinical implications. Previous studies have indicated that TPMT activity in ALL is inversely associated with TGNs concentration and overall cytotoxic effect of thiopurines, while the risk of relapse was demonstrated to be lower for patients with low TPMT activity levels $(3,9,15)$. High TPMT activity in cell lines and in patients with IBD was suggested to be associated with thiopurine resistance and a high methylMP/TGNs ratio (25). Escalation of thiopurine dose for these patients is ineffective due to a preferential methylMP production $(25,34)$. Maintenance chemotherapy in pediatric AML has been demonstrated to be a subject of controversy: No benefit was indicated in several studies (35); however, it is assumed that it may have positive effect in subgroups of patients currently emerging from new risk-stratification AML therapy (35). 6-TG-based maintenance chemotherapy is being used in the 
AML-BFM 2012 Protocol (36) for children and adolescents assigned to standard and intermediate risk groups according to molecular genetic factors.

A small sample size with a heterogeneous structure in terms of AML subtypes and other risk factors, including molecular and cytogenetic data, were not always available, making it difficult to draw comprehensive conclusions from the data of the present study. An additional limitation is that different children with AML were included in each of the groups: At the moment of diagnosis; during the maintenance therapy; and following the cessation of the chemotherapy. A 19.7-year old patient was included to the study group as he was diagnosed with AML in the pediatric center at the age of 18 , and the TPMT evaluation took place following chemotherapy. According to previous studies, children demonstrate almost equivalent levels of TPMT activity compared with adults (37).

The preliminary results of the present study suggest that TPMT activity in patients with AML may be increased compared with those in patients with ALL. Additional studies on thiopurine metabolism in AML are required to confirm this, and to clarify the clinical implications of the associations with treatment outcome, and with regard to the cytogenetic and molecular factors currently used for AML risk stratification.

\section{Acknowledgements}

Not applicable.

\section{Funding}

No funding was received.

\section{Availability of data and materials}

The datasets used and/or analyzed during the current study are available from the corresponding author on reasonable request.

\section{Authors' contributions}

JW designed the present study; JS and JSS wrote the manuscript; and MC and JW revised the manuscript. JS, JSS, MC, MR, SK, MW and JW contributed to the acquisition, analysis and interpretation of the data and have seen and approved the final manuscript.

\section{Ethics approval and consent to participate}

The study was approved by the Bioethical Committee at Poznan University of Medical Sciences (Poznań, Poland), was performed in accordance with the 1964 Declaration of Helsinki and its later amendments. Written informed consent was obtained from the parents of the patients prior to initiating the study.

\section{Consent for publication}

Patients consent for the publication their data was received.

\section{Competing interests}

The authors declare that they have no competing interests.

\section{References}

1. de Rooij JD, Zwaan CM and van den Heuvel-Eibrink M: Pediatric AML: From biology to clinical management. J Clin Med 4: 127-149, 2015.

2. Tarlock K and Meshinchi S: Pediatric acute myeloid leukemia: Biology and therapeutic implications of genomic variants. Pediatr Clin North Am 62: 75-93, 2015.

3. Stensman LM, Kjeldsen E, Nersting J, Schmiegelow K and Hasle $\mathrm{H}$ : Treatment-related myelodysplastic syndrome in a child with acute myeloid leukemia and TPMT heterozygosity. J Pediatr Hematol Oncol 37: e242-e244, 2015.

4. Pui CH, Carroll WL, Meshinchi S and Arceci RJ: Biology, risk stratification, and therapy of pediatric acute leukemias: An update. J Clin Oncol 29: 551-565, 2011.

5. Mussai FJ, Yap C, Mitchell C and Kearns P: Challenges of clinical trial design for targeted agents against pediatric leukemias. Front Oncol 4: 374, 2015.

6. Creutzig U, Zimmermann M, Bourquin JP, Dworzak MN, Fleischhack G, Graf N, Klingebiel T, Kremens B, Lehrnbecher T, von Neuhoff C, et al: Randomized trial comparing liposomal daunorubicin with idarubicin as induction for pediatric acute myeloid leukemia: Results from study AML-BFM 2004. Blood 122: 37-43, 2013.

7. Munshi PN, Lubin M and Bertino JR: 6-thioguanine: A drug with unrealized potential for cancer therapy. Oncologist 19: 760-765, 2014.

8. Pizzo PA and Poplack DG: Principles and practice of pediatric oncology. Philadelphia, Lippincott Williams and Wilkins, pp309, 2011.

9. Coulthard S and Hogarth L: The thiopurines: An update. Invest New Drugs 23: 523-532, 2005.

10. Coulthard SA, Howell C, Robson J and Hall AG: The relationship between thiopurine methyltransferase activity and genotype in blasts from patients with acute leukemia. Blood 92: 2856-2862, 1998.

11. Katara P and Kuntal H: TPMT polymorphism: When shield becomes weakness. Interdiscip Sci 8: 150-155, 2016.

12. Lennard L and Lilleyman JS: Individualizing therapy with 6-mercaptopurine and 6-thioguanine related to the thiopurine methyltransferase genetic polymorphism. Ther Drug Monit 18: 328-334, 1996.

13. Abaji R and Krajinovic M: Thiopurine S-methyltransferase polymorphisms in acute lymphoblastic leukemia, inflammatory bowel disease and autoimmune disorders: Influence on treatment response. Pharmgenomics Pers Med 10: 143-156, 2017.

14. Schmiegelow K, Forestier E, Kristinsson J, Söderhäll S, Vettenranta K, Weinshilboum R and Wesenberg F; Nordic Society of Paediatric Haematology and Oncology: Thiopurine methyltransferase activity is related to the risk of relapse of childhood acute lymphoblastic leukemia: Results from the NOPHO ALL-92 study. Leukemia 23: 557-564, 2009.

15. Lennard L, Cartwright CS, Wade R and Vora A: Thiopurine methyltransferase and treatment outcome in the UK acute lymphoblastic leukaemia trial ALL2003. Br J Haematol 170: 550-558, 2015 .

16. Tamm R, Mägi R, Tremmel R, Winter S, Mihailov E, Smid A, Möricke A, Klein K, Schrappe M, Stanulla M, et al: Polymorphic variation in TPMT is the principal determinant of TPMT phenotype: A meta-analysis of three genome-wide association studies. Clin Pharmacol Ther 101: 684-695, 2017.

17. Stary J, Zimmermann M, Campbell M, Castillo L, Dibar E, Donska S, Gonzalez A, Izraeli S, Janic D, Jazbec J, et al: Intensive chemotherapy for childhood acute lymphoblastic leukemia: Results of the randomized intercontinental trial ALL IC-BFM 2002. J Clin Oncol 32: 174-184, 2014.

18. Chrzanowska M, Kuehn M, Januszkiewicz-Lewandowska D, Kurzawski M and Droździk M: Thiopurine S-methyltransferase phenotype-genotype correlation in children with acute lymphoblastic leukemia. Acta Pol Pharm 69: 405-410, 2012.

19. Kröplin T, Weyer N, Gutsche S and Iven $H$ : Thiopurine S-methyltransferase activity in human erytrocytes: A new HPLC method using 6-thioguanine as substrate. Eur J Clin Pharmacol 54: 265-271, 1998.

20. Kröplin T and Iven H: Methylation of 6-mercaptopurine and 6-thioguanine by thiopurine S-methyltransferase. A comparison of activity in red blood cell samples of 199 donors. Eur J Clin Pharmacol 56: 343-345, 2000. 
21. Kurzawski M, Gawrońska-Szklarz B and Droździk M: Frequency distribution of thiopurine S-methyltransferase alleles in a polish population. Ther Drug Monit 26: 541-545, 2004.

22. Kotur N, Dokmanovic L, Janic D, Stankovic B, Krstovski N, Tosic N, Katsila T, Patrinos GP, Zukic B and Pavlovic S: TPMT gene expression is increased during maintenance therapy in childhood acute lymphoblastic leukemia patients in a TPMT gene promoter variable number of tandem repeat-dependent manner. Pharmacogenomics 16: 1701-1712, 2015.

23. Chouchana L, Narjoz C, Roche D, Golmard JL, Pineau B, Chatellier G, Beaune P and Loriot MA: Interindividual variability in TPMT enzyme activity: 10 years of experience with thiopurine pharmacogenetics and therapeutic drug monitoring. Pharmacogenomics 15: 745-757, 2014

24. Anasari A, Hassan C, Duley J,Marinaki A, Shobowale-Bakre EM, Seed P, Meenan J, Yim A and Sanderson J: Thiopurine methyltransferase activity and the use of azathioprine in inflammatory bowel disease. Aliment Pharmacol Ther 16: 1743-1750, 2002.

25. Chouchana L, Narjoz C, Beaune P, Loriot MA and Roblin X: Review article: The benefits of pharmacogenetics for improving thiopurine therapy in inflammatory bowel disease. Aliment Pharmacol Ther 35: 15-36, 2012.

26. Karas-Kuzelicki N and Mlinaric-Rascan I: Individualization of thiopurine therapy: Thiopurine S-methyltransferase and beyond Pharmacogenomics 10: 1309-1322, 2009.

27. Roberts RL, Gearry RB, Bland MV, Sies CW, George PM, Burt M, Marinaki AM, Arenas M, Barclay ML and Kennedy MA: Trinucleotide repeat variants in the promoter of the thiopurine S-methyltransferase gene of patients exhibiting ultra-high enzyme activity. Pharmacogenet Genomics 18: 434-438, 2008.

28. Chouchana L, Roche D, Jian R, Beaune P and Loriot MA: Poor response to thiopurine in inflammatory bowel disease: How to overcome therapeutic resistance? Clin Chem 59: 1023-1026, 2013.

29. Stocco G, Yang W, Crews KR, Thierfelder WE, Decorti G, Londero M, Franca R, Rabusin M, Valsecchi MG, Pei D, et al: PACSIN2 polymorphism influences TPMT activity and mercaptopurine-related gastrointestinal toxicity. Hum Mol Genet 21: 4793-4804, 2012.
30. Smid A, Karas-Kuzelicki N, Jazbec J and Mlinaric-Rascan I: PACSIN2 polymorphism is associated with thiopurine-induced hematological toxicity in children with acute lymphoblastic leukaemia undergoing maintenance therapy. Sci Rep 6: 30244 2016.

31. Karas-Kuželički N, Šmid A, Tamm R, Metspalu A and Mlinarič-Raščan I: From pharmacogenetics to pharmacometabolomics: SAM modulates TPMT activity. Pharmacogenomics 15: 1437-1449, 2014

32. Milek M, Karas Kuzelicki N, Smid A and Mlinaric-Rascan I: S-adenosylmethionine regulates thiopurine methyltransferase activity and decreases 6-mercaptopurine cytotoxicity in MOLT lymphoblasts. Biochem Pharmacol 77: 1845-1853, 2009.

33. Lennard L, Cartwright CS, Wade R, Richards SM and Vora A: Thiopurine methyltransferase genotype-phenotype discordance and thiopurine active metabolite formation in childhood acute lymphoblastic leukaemia. Br J Clin Pharmacol 76: 125-136, 2013.

34. Chouchana L, Fernández-Ramos AA, Dumont F, Marchetti C, Ceballos-Picot I, Beaune P, Gurwitz D and Loriot MA: Molecular insight into thiopurine resistance: Transcriptomic signature in lymphoblastoid cell lines. Genome Med 7: 37, 2015.

35. Gibson B, Perentesis J, Alonzo TA and Kaspers GL: Treatment of Acute Myeloid Leukemia. In: Childhood Leukemia Springer-Verlag Berlin Heidelberg, pp136-137, 2011.

36. https://www.clinicaltrialsregister.eu/ctr-search/trial/2013-000018-39/ DE (entry date 20 April 2018.)

37. Asadov C, Aliyeva G and Mustafayeva K: Thiopurine S-methyltransferase as a pharmacogenetic biomarker: Significance of testing and review of major methods. Cardiovasc Hematol Agents Med Chem 15: 23-30, 2017. 